\title{
Kassenpleite
}

\section{Vorsicht bei freiwilligen Leistungen}

\author{
Grundsätzlich müssen Vertragsärzte eine Insolvenz einer Kranken- \\ kasse, wie jetzt etwa die der City BKK, nicht fürchten. Sie genießen \\ rechtlich eine privilegierte Position und können ihre Ansprüche \\ voll geltend machen. Vorsicht ist allerdings bei freiwilligen Leistungen \\ der Pleitekasse geboten. Diese muss die neue Krankenkasse nicht \\ zwangsläufig übernehmen.
}

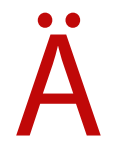
rzte sind im Sozialsystem zur Leistungserbringung verpflichtet auch gegenüber Patienten, deren Krankenkasse sich in der Auflösung befindet. Aber werden ihre Leistungen im Fall der Insolvenz auch vergütet?

Nachdem die Bundesländer nach Einführung des Gesetzes zur Weiterentwicklung der Organisationsstruktur in der gesetzlichen Krankenversicherung (GKVOrgWG) nicht mehr in der Haftung stehen, ist nunmehr im 5. Sozialgesetzbuch (SGB V) festgelegt, wer für die Altverbindlichkeiten insolventer Krankenkassen haftet ( $\$ 171 \mathrm{~d}$ Abs. 5 sowie $\$ 155$ Abs. 5 Satz 1 Nr. 3 und 5). Das ist auch dringend nötig. Denn ein Kassenarzt ist Leistungserbringer im öffentlich-rechtlichen System der GKV und muss auf die Leistungsfähigkeit der Krankenkassen vertrauen können. Er ist in seiner finanziellen Existenz davon abhängig, dass die Kassen - als juristische Personen des öffentlichen Rechts unmittelbar dem Grundgesetz unterworfen - seine Vergütungsansprüche begleichen. Letzteres gilt insbesondere, wenn er außerhalb des Systems der GKV unmittelbar mit einer Krankenkasse im Rahmen von IV-Verträgen abrechnet. Daher ist er auch durch Artikel 12 Grundgesetz (Artikel zur Berufsfreiheit) im Vergleich zu anderen Gläubigern der Krankenkasse mit einer verfassungsrechtlich besonders abgesicherten Rechtsposition ausgestattet. Gleiches gilt entsprechend gegenüber den KVen. Folgerichtig regeln die einschlä- gigen Paragrafen im SGB V, dass der Arzt sich nicht auf eine quotale Befriedigung ( $\$ 195$ Insolvenzordnung) verweisen lassen muss, sondern seine Ansprüche privilegiert in voller Höhe geltend machen kann.

\section{So sieht die Regelung aus}

Bei einem Schwellenwert von bis zu einem Prozent des jährlichen Gesamtbetrages, den die Krankenkassen der jeweiligen Kassenart als Zuweisung aus dem Gesundheitsfonds erhalten, haften die verbleibenden Krankenkassen der Kassenart. Für Verpflichtungen oberhalb des Schwellenwertes haften auch die Krankenkassen der übrigen Arten. Für Kassenärzte ist daher grundsätzlich kein Forderungsausfall zu erwarten. Die Ärzte mögen sich in diesem Zusammenhang vergegenwärtigen, dass das KV-System aufgrund dieser Sicherheit seine Vorteile hat. Im Insolvenzfall einer Kasse ist mit der Haftungsgemeinschaft der Krankenkassen ein leistungsfähiger Schuldner vorhanden, sodass dem Arzt sein voller Honoraranspruch erhalten bleibt. Dies gilt auch für Leistungen, die gegenüber der insolventen Krankenkasse noch im Rahmen von IVVerträgen erbracht wurden.

Die Erfüllung der noch ausstehenden Forderungen wird ab dem Zeitpunkt der Eröffnung des Insolvenzverfahrens zentral durch den GKV-Spitzenverband übernommen, der dann bei den betreffenden Krankenkassen Regress nimmt. Abzu-

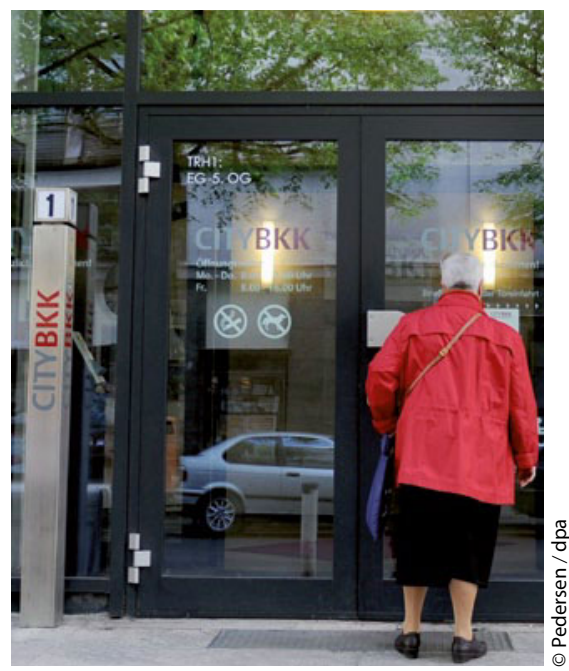

Aktuelles Beispiel für eine Kassenpleite: die City-BKK

rechnen sind die Leistungen weiterhin gegenüber den KVen.

\section{Kostenübernahme bei \\ Zusatzleistungen nicht gesichert}

Vor allem im Bereich freiwilliger Leistungen sind jedoch Schwierigkeiten vorprogrammiert, denn diese unterscheiden sich von Kasse zu Kasse erheblich. Wechselt der Patient während der Behandlung in die neue Krankenkasse, ist diese nicht zwangsläufig verpflichtet, die freiwilligen Leistungen $\mathrm{zu}$ übernehmen. Folglich müsste gegebenenfalls direkt gegenüber dem Patienten abgerechnet werden. Ärzten und auch Patienten ist daher zu empfehlen, sich über Zusatzleistungen, die die insolvente Krankenkasse angeboten hat, $\mathrm{zu}$ informieren. Um nicht auf den Kosten sitzen zu bleiben, bietet es sich an, eine gesonderte Kostenübernahmebelehrung und -vereinbarung mit dem Patienten für den Fall zu treffen, dass die Krankenkasse die Kostenübernahme ablehnt. Weiter kann es sich in einzelnen Fällen anbieten, sich eines Kostenvoranschlages zu bedienen. Liegt hierfür bereits eine Genehmigung der insolventen Krankenkasse vor, so ist auch die Krankenkasse, in die der Patient nach dem Insolvenzfall wechseln wird, an die Leistungserbringung gebunden. RA Heike Jablonsky, RA Nina Graap

Heike Jablonsky ist Fachanwältin für Medizinrecht und Arbeitsrecht in Celle

www.ra-jablonsky.de 